\title{
A molecular dynamics study on glucose
}

\author{
molecular recognition by a non-
}

enzymatic selective sensor based on a

\section{conducting polymer}

David Zanuy, 1,* Georgina Fabregat, $^{1,2}$ Carlos A. Ferreira ${ }^{3}$ and Carlos Alemán ${ }^{1,2, *}$

${ }^{1}$ Departament d'Enginyeria Química, EEBE, Universitat Politècnica de Catalunya, C/ Eduard Maristany 10-14, Ed. I2, 08019 Barcelona, Spain

${ }^{2}$ Barcelona Research Center for Multiscale Science and Engineering, Universitat Politècnica de Catalunya, Eduard Maristany 10-14, 08019 Barcelona, Spain

${ }^{3}$ Universidade Federal do Rio Grande do Sul - DEMAT - Av. Bento Gonçalves, 9500 setor 4- prédio 43426 - Cep. 91501-970 - Porto Alegre - RS - Brazil.

* david.zanuy@upc.edu and carlos.aleman@upc.edu. 


\begin{abstract}
Poly(hydroxymethyl-3,4-ethylendioxythiophene) (PHMeDOT), a very electroactive polythiophene derivative bearing a dioxane ring fused onto the thiophene ring and an exocyclic hydroxymethyl substituent, is able to electrocatalyze the oxidation of glucose in the presence of interferents (e.g. dopamine, uric acid and ascorbic acid) without the assistance of an enzymatic catalyst. In this work, after demonstrating that the chronoamperometric response of such polythiophene derivatives allows discrimination of glucose from fructose, the $\mathrm{PHMeDOT} \cdot$ ' sugar recognition mechanism has been investigated using atomistic computer simulations. More specifically, molecular dynamics simulations were conducted on model systems formed by a steel surface covered with a nanometric film of PHMeDOT, which was immersed in an aqueous environment with a few explicit sugar molecules (i.e. glucose or fructose). Analyses of the trajectories indicate that glucose interacts with PHMeDOT forming a well-defined network of specific hydrogen bonds. More specifically, glucose prefers to interact as a hydrogen bonding donor using the hydroxyl group tether to the main sugar ring, while PHMeDOT acts as the hydrogen bonding acceptor. Interestingly, (glucose) $\mathrm{O}-\mathrm{H}^{*} \cdot{ }^{\cdot \mathrm{O}(\mathrm{PHMeDOT})}$ interactions involve, as hydrogen bonding acceptors, not only the oxygen atoms of the dioxane ring but also the oxygen atom of the exocyclic hydroxymethyl substituent, which is a differential trend with respect to the other polythiophene derivatives that do not exhibit sensing ability. In contrast, fructose does not present such well-defined patterns of specific interactions, especially those that are distinctive because of the exocyclic hydroxymethyl substituent, making the experimental observations understandable.
\end{abstract}




\section{INTRODUCTION}

The incidence of diabetes, which is caused by the inadequate production of insulin by the pancreas or the inability to effectively utilize insulin, has steadily increased in recent decades. In $20158.8 \%$ of adults between the ages of 20 and 79 years were diabetic and the International Diabetes Federation (IDF) has predicted that the number of diabetes patients will increase to $10.4 \%$ of the world's population and will be the seventh-leading cause of mortality in $2040 .{ }^{1}$ It is well-known that strict sugar monitoring can improve the survival of diabetes patients and prevent complications related to this disease. ${ }^{2-4}$ Most glucose monitors use an enzyme-based electrochemical sensors, ${ }^{5-10}$ many of them being currently commercialized and marketed by global corporations. Glucose oxidase (GOx) and glucose dehydrogenase are the most commonly used enzymes in this technology. ${ }^{11,12}$ However, these sensors are seriously influenced by the environmental conditions (i.e. $\mathrm{pH}$, humidity and temperature), which may affect the enzyme activity. In particular temperature above $40{ }^{\circ} \mathrm{C}$, high or low humidity, and $\mathrm{pH}$ values below 2 and above 8 , can cause severe damages to the enzyme.

As sensitivity of glucose sensors largely depends on the activity of the immobilized enzyme, non-enzymatic sensors without biological functional units are considered advantageous in terms of structural simplicity and quality control for mass production. Within this context, non-biological materials have attracted significant attention as electrodes during the last years, ${ }^{13-16}$ offering a number of opportunities to materials scientists. For example, non-enzymatic sensors based on nanoporous platinum can be liberated from the constraints associated to temperature, humidity, solvent and processes in manufacturing. ${ }^{17,18}$ Nanostructures based on other metals, as for example gold $^{19,20}$ and nickel, ${ }^{21}$ and alloys ${ }^{22-24}$ have been also successfully employed for glucose detection. Besides, molecularly-imprinted polymers have been used as artificial molds 
to chemically mimicking the host-guest molecular interactions found in glucose-enzyme complexes by introducing functional moieties, such as amine, carboxyl and hydroxyl. ${ }^{25,26}$

Conducting polymers (CPs) have been also used to fabricate non-enzymatic glucose sensors. In particular, CPs have been combined with inorganic catalysts (i.e. metals or metal oxides), which facilitate the electroxidation of glucose that is kinetically very slow. ${ }^{27-29}$ In addition, some CPs have also been found to detect glucose without the assistance of any catalytic agent. ${ }^{30,31}$ Within this context, we recently reported the electrochemical detection of glucose using a CP that is based on nanometric films of a very electroactive poly(3,4-ethylenedioxythiophene) (PEDOT) derivative that incorporates a hydroxyl substituent per repeat unit, poly(hydroxymethyl-3,4ethylendioxythiophene) (PHMeDOT in Scheme 1). ${ }^{31}$ The sensor, which is enzyme free and does not require from additional catalytic nanoparticles, showed excellent tolerance against interferents, a low detection limit, and a deviation lower than $2 \%$ with respect to measures in human blood samples with commercial sensors. Although the response of this CP was tentatively attributed to the closeness between the hydroxyl substituent and the aromatic ring of the rigid backbone, ${ }^{31}$ the detection mechanism remains unknown.

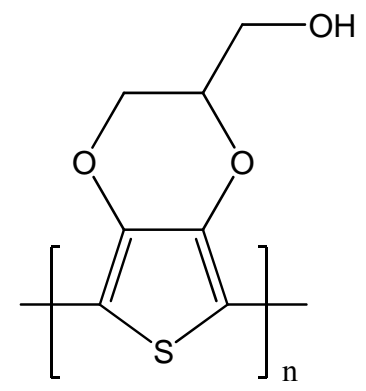

Scheme 1: Chemical structure of PHMeDOT

The main focus of this work is to provide a comprehensive picture of the sensing mechanism for the glucose detection using PHMeDOT. Thus, after experimental 
demonstration of the selectivity of PHMeDOT for glucose sensing towards fructose, atomistic Molecular Dynamics (MD) simulations have been conducted on PHMeDOT $\cdots$ sugar complexes considering both glucose and fructose. More specifically, a modelling approach has been applied to examine the topology and dynamical characteristics of PHMeDOT $\cdots$ sugar intermolecular interactions and, thus, establish the interaction pattern necessary for the successful recognition of glucose.

\section{METHODS}

\section{Experimental methods}

Materials. Thieno[3,4-b]-1,4-dioxin-2-methanol (HMeDOT) monomer, anhydrous lithium perchlorate $\left(\mathrm{LiClO}_{4}\right), \mathrm{D}$-glucose and D-fructose of analytical reagent grade were purchased from Sigma-Aldrich (Spain). All chemicals were used without further purification. Anhydrous $\mathrm{LiClO}_{4}$ was stored in an oven at $80{ }^{\circ} \mathrm{C}$ before use in the electrochemical trials. Phosphate buffer solution (PBS) $0.1 \mathrm{M}$ with $\mathrm{pH}=7.4$ was prepared as electrolyte solution by mixing four stock solutions of $\mathrm{NaCl}, \mathrm{KCl}, \mathrm{NaHPO}_{4}$ and $\mathrm{KH}_{2} \mathrm{PO}_{4}$.

Synthesis. PHMeDOT films were produced by chronoamperometry (CA) under a constant potential of $0.80 \mathrm{~V}$. Stainless steel AISI 316 sheets of $0.25 \times 0.25 \mathrm{~cm}^{2}$ was used as working and counter electrodes. The reference electrode was an $\mathrm{Ag} \mid \mathrm{AgCl}$ electrode. Films were obtained using a $0.1 \mathrm{M}$ monomer aqueous solution with $0.1 \mathrm{M} \mathrm{LiClO}_{4}$ and employing a polymerization time of $10 \mathrm{~s}$. All electrochemical experiments were conducted on a PGSTAT302N AUTOLAB potenciostat-galvanostat (Ecochimie, The Netherlands) equipped with the ECD module to measure very low current densities (100 $\mu$ A-100 pA), which was connected to a PC computer controlled through the GPES software. 
Electrochemical detection. Chronoamperometric measurements were carried out at room temperature in the reaction cell containing $100 \mu \mathrm{L}$ of $0.1 \mathrm{M}$ PBS at a polarization potential of $-600 \mathrm{mV}$ using the Autolab PGSTAT302N equipment described above. Glucose and fructose solutions were prepared in $10 \mathrm{mM}$ PBS and allowed to mutarotate overnight. Choroamperometric curves were obtained after adding $4 \mu \mathrm{L}$ of a $25 \mathrm{mM}$ glucose or fructose solution (i.e. the effective sugar concentration was $1 \mathrm{mM}$ ) under constant stirring at $100 \mathrm{~s}$ intervals.

\section{Theoretical methods}

Force-field parametrization. The doping level reported for PHMeDOT is +0.9 per repeat unit. ${ }^{31}$ This value is significantly higher than the value of +0.5 per repeat unit obtained for PEDOT, ${ }^{32}$ which explained the noticeably high electrochemical activity of PHMeDOT. ${ }^{31}$ In this work, atomic charges for PHMeDOT repeat unit (Figure S1) were computed considering a doping level of +1.0 and using the Restrained ElectroStatic Potential (RESP) strategy. ${ }^{33}$ Stretching, bending, torsional and van der Waals force-field parameters for PHMeDOT were extracted from previous studies on PEDOT $^{34}$ in which a complete set of parameters for PEDOT was adapted to the $\mathrm{AMBER}^{35}$ force field. Force-field parameters for perchlorate $\left(\mathrm{ClO}_{4}^{-}\right)$and iron were previously reported by Zanuy and Alemán, ${ }^{36}$ while those describing glucose and fructose were directly extracted from GLYCAM AMBER libraries. ${ }^{37}$ Finally, water molecules were described using TIP3 model. ${ }^{38}$

Molecular model. Firstly, a representative molecular model of the bulk organization of the PHMeDOT coated steel surface was built using our new implemented algorithm to represent topographic and physical characteristic of $\mathrm{CP}$ chains grown onto flat 
inorganic electrodes. ${ }^{36,39}$ In order to reach the experimental thickness (i.e. $\sim 90 \mathrm{~nm}$, as described below), a PHMeDOT model with 14000 repeat units was grown onto a surface of $7.665 \times 7.665 \mathrm{~nm}^{2}$ made of 8126 iron atoms arranged in a FCC cell. ${ }^{36}$ Polymer chains were generated using a modified Metropolis criterion analogous to that employed in the ConRot method. ${ }^{40}$ Thus, the acceptance probability was not directly assessed by the energy difference of the starting and final states but by computing the probability of each individual change (i.e. the addition of a repeat unit to a given chain). ${ }^{36,39}$ Iron atoms were kept fixed at their equilibrium positions in all simulations (see below) since from a practical stand point these atoms do not directly participate in the sugar $\cdots$ PHMeDOT recognition process.

After reaching the desired polymer thickness, the whole model was completed by inserting randomly $14000 \mathrm{ClO}_{4}^{-}$molecular anions within the $\mathrm{CP}$ matrix, which acted as doping ions. As only non-bonding energy contributions were employed to build the bulk polymer organization, the system was relaxed using 3500 steps of energy minimization using NAMD $2.12^{41}$ and AMBER. ${ }^{35}$ After this, the relaxed molecular model was then submerged in a previously equilibrated water box of $7.665 \times 7.665 \times 150.0 \mathrm{~nm}^{3}$. Any water molecule that overlapped with any of the atoms belonging to the solid bulk was removed and, finally, a total of 76170 water molecules were kept. Accordingly, the system used for model the sugar detection by PHMeDOT presented 433466 explicit atoms. Details about the procedure used to construct the model were provided in our recent studies. $^{36,39}$

In order to correct the distribution of solvent molecules along the z-direction, the density was equilibrated by $6.5 \mathrm{~ns}$ of asymmetric NPT-MD, in which only the zdimension was allowed to vary $\left(\mathrm{NP}_{z} \mathrm{~T}\right.$ conditions), before run the corresponding production trajectories. Figure S2 shows the evolution of $c$-parameter with simulated 
time until reaches a steady state at $\sim 105.5 \AA$. Details of the protocol used for the equilibration of the simulation box are given in the next sub-section.

Once a stable biphasic model (i.e. a solid bulk polymer over a metallic surface and water phase on top of it embedding all the interphase polymer chains) was obtained, 3 molecules of glucose or 3 molecules of fructose were inserted at the same positions: right on the top of the larger polymer chain tips. After a quick re-equilibration (see next sub-section), production trajectories with glucose- and fructose-containing models were $30 \mathrm{~ns}$ each. In order to ensure our results reproducibility, additional production MD simulations were performed using independent models, which were obtained using the same procedures described above but introducing small changes in both the polymer growth and the solvation steps.

Equilibrations of the simulation box and production simulations. After the distribution of solvent molecules along the z-direction, the total energy of the system was again optimized with 5000 steps more of energy minimization. Then, we proceeded to optimize the system's density in two steps:

i) The solvent bulk was equilibrated by two consecutive MD runs. First, the temperature of the solvent was brought to $298 \mathrm{~K}$ and equilibrated at this value using $1.5 \cdot 10^{5}$ steps of NVT-MD and applying the Berendesen Barostat. ${ }^{42}$ Second, $0.5 \cdot 10^{6}$ steps of $\mathrm{NP}_{z} \mathrm{~T}-\mathrm{MD}$ were conducted using a combination of the Nose-Hoover piston ${ }^{43}$ with the piston fluctuation control of temperature implemented for Langevin Dynamics. $^{44}$ Pressure was kept at 1.01325 bars while the oscillation period and the piston decay time were set at $1 \mathrm{ps}$ and $0.001 \mathrm{ps}$, respectively. The piston temperature was set at the same value as the thermostat control, 298K, which used a damping coefficient of 2 ps. In the $\mathrm{NP}_{z} \mathrm{~T}$ ensemble, only the box length in the $z$-direction was 
allowed to change, where the z-component of the pressure tensor is equal to the external pressure. $^{45,46}$

ii) Once the solvent was equilibrated, all the polymer atoms were liberated and brought to the target temperature by $1.0 \cdot 10^{5}$ steps of NVT-MD using the Langevin temperature control, in which the thermostat control temperature was set at $298 \mathrm{~K}$ with a damping coefficient of $2 \mathrm{ps}$. This last mini-run gave way to $6.5 \cdot 10^{6}$ steps of $\mathrm{NP}_{z} \mathrm{~T}-\mathrm{MD}$ until stable box dimensions were reached (Figure S2), using the same exact protocol exposed above for the water phase equilibration.

After the addition of the three sugar molecules, a quick new equilibration consisting on $1.5 \cdot 10^{5}$ steps of $\mathrm{NP}_{z} \mathrm{~T}-\mathrm{MD}$ was set, which led to the starting points of the production runs. All the MD runs conducted after glucose or fructose incorporation used the same conditions described above for the box size equilibration run.

In all MD runs, the time step was set to $1 \mathrm{fs}$ and the non-bonding list was updated every 10 steps. Periodic boundary conditions were applied using the nearest image convention and the atom pair cut-off distance used to compute the van der Waals interactions was set at $14.0 \AA$. In order to avoid discontinuities in the potential energy function, non-bonding energy terms were forced to slowly converge to zero, by applying a smoothing factor from a distance of 12.0 Å. Beyond cut off distance, electrostatic interactions were calculated by using Particle Mesh of Ewald, with a points grid density of the reciprocal space of $1 \AA^{3}{ }^{47}$

\section{RESULTS AND DISCUSSION}

The globular morphology of PHMeDOT nanofilms (90 $\pm 5 \mathrm{~nm}$ in thickness) prepared using a polymerization time of $10 \mathrm{~s}$ is displayed in Figure S3. The current-time response of as prepared PHMeDOT was examined by applying a polarization potential of -600 
$\mathrm{mV}$ vs $\mathrm{Ag} \mid \mathrm{AgCl}$ to a $0.1 \mathrm{M}$ PBS stirred solution. For this purpose, $4 \mu \mathrm{L}$ of a $25 \mathrm{mM}$ glucose solution were injected into the PBS-containing electrochemical cell, representing an effective glucose concentration of only $1 \mathrm{mM}$ (i.e. the concentration of glucose in human blood is between 4.4 and $6.6 \mathrm{mM}) .{ }^{48}$ Figure 1a shows the successful amperometric response of PHMeDOT with successive additions of glucose, while the performance of the sensor to differentiate glucose from fructose is demonstrated in Figure $1 \mathrm{~b}$. It can be seen that, although the addition of $1 \mathrm{mM}$ fructose (i.e. $4 \mu \mathrm{L}$ of a 25 $\mathrm{mM}$ fructose solution) also increased the current of PHMeDOT electrode, the intensity increased was much smaller than that caused by the addition of $1 \mathrm{mM}$ glucose. Accordingly, PHMeDOT exhibits high selectivity for glucose sensing not only towards dopamine, uric acid and ascorbic acid, as demonstrated in previous work, ${ }^{31}$ but also towards fructose.

After prove the selectivity of PHMeDOT for glucose towards fructose, atomistic MD simulations of complexes formed by a $\mathrm{CP}$ film tethered to a steel surface and explicit glucose or fructose molecules in an aqueous environment were performed. In order to identify the formation specific interactions and, therefore, to ascertain the PHMeDOT $\cdots$ glucose recognition pattern, a general analysis of all possible intermolecular interaction modes of the studied sugars was initially conducted. For this purpose, the radial distributions of pair distances, $g(r)$, between the mass center of the sugar molecules and the mass centers of the PHMeDOT repeat units or the $\mathrm{ClO}_{4}^{-}$ counterions were calculated for each production trajectory.

Figure 2 compares the $g(r)$ profiles obtained for the mass centers of the PHMeDOT repeat units and the $\mathrm{ClO}_{4}^{-}$counterions. As it can be seen, the areas of the radial distributions calculated for the PHMeDOT repeat units are overestimated with respect to those derived from the $\mathrm{ClO}_{4}{ }^{-}$counterions, independently of the sugar. This is because 
the $\mathrm{g}(r)$ profiles were not computed for all $14000 \mathrm{CP}$ repeat units but only for those below a cutoff distance of $14 \AA$, while all the counterions were considered in the corresponding $\mathrm{g}(r)$ profiles. This different treatment is shown to magnify the differences found between the two sugars in the former $g(r)$ profiles. Thus, it is quite clear that the $\mathrm{ClO}_{4}{ }^{-}$profiles do not show any differential behavior when interacting with glucose and fructose (Figure $2 \mathrm{a}$ and $2 \mathrm{~b}$, respectively). In contrast, significant differences are found in the profiles calculated with respect to the PHMeDOT repeat units. More specifically, glucose only seems to associate with the $\mathrm{CP}$ by a unique distance, which is slightly above $5 \AA$, whereas fructose shows a small peak below $5.0 \AA$ and a higher peak above $5.0 \AA$ A. These geometrical differences in the interaction distance would hold the key to understand the different experimental behavior observed when the $\mathrm{CP}$ associate with either glucose or fructose. According to these results, more detailed analyses have been focused on PHMeDOT $\cdots$ sugar specific interactions considering the three explicit molecules included in the simulation box for each kind of sugar.

The potential hydrogen bond donors and acceptor of the two sugars and PHMeDOT repeat units are labeled in Figure 3a, while the distributions of distances between the hydroxyl hydrogen atom of PHMeDOT repeat units (i.e. hydrogen bonding donor: H$\mathrm{OM}$ in Figure 3a) and the oxygen atoms of glucose and fructose (i.e. hydrogen bonding acceptors: O\# with \# ranging from 1 to 6 in Figure 3a), are displayed in Figures $3 \mathrm{~b}$ and $3 \mathrm{c}$, respectively. As it can be seen, the ability of the $\mathrm{CP}$ of interacting as hydrogen bonding donor with the oxygen atoms of sugars is practically null, suggesting that PHMeDOT and sugars preferably acts as hydrogen bonding acceptor and donors, respectively. This hypothesis is corroborated in Figure 4, which shows the distribution of distances between the hydroxyl hydrogen atoms of glucose or fructose and the OE and $\mathrm{OM}$ oxygen atoms of the $\mathrm{CP}$ repeat units. 
The distributions of distances depicted in Figure 4 indicate that glucose exhibit a major propensity to be closer to the dioxane oxygen atoms of PHMeDOT than fructose. This is clearly reflected by the almost homogeneous radial distributions of distance obtained between any hydrogen bond donor of glucose and the OE hydrogen bonds acceptors of PHMeDOT. Thus, the five $\mathrm{OE} \cdots \mathrm{H}-\mathrm{O} \#$ distributions displayed in Figure $4 \mathrm{a}$ shows a well-defined peak centered at $\sim 3.5 \AA$. Although the latter distance is actually larger than that expected for strong hydrogen bonds, the presence of such peak clearly allows us to identify the formation of (PHMeDOT)OE $\cdots \mathrm{H}-\mathrm{O}($ glucose) specific interactions. Inspection of the distribution profiles obtained for fructose (Figure $4 b$ ) indicates that, among all potential hydrogen bond donors, only $\mathrm{H}-\mathrm{O} 5$ shows a peak centered at $3.75 \AA$ that is consistent with the formation of specific interactions with the oxygen atoms of the $\mathrm{CP}$ dioxane ring. Finally, inspection of the distributions of distances involving the exocyclic hydroxyl group of PHMeDOT, which are included in Figure 4, reveals that $\mathrm{OM} \cdots \mathrm{H}-\mathrm{O}$ (sugar) interactions are practically inexistent.

Overall, results displayed in Figures 3 and 4 shows significant difference between fructose and glucose. The former exhibits a reduced the ability to form an interaction network when associated with polymer chains, while the latter forms well-defined hydrogen bonding interactions with the CP. The multiple PHMeDOT $\cdots$ glucose specific interactions enables the most favorable orientation of that sugar molecule with respect to the CP chains, which apparently is not achieved by fructose. As mentioned above, plots displayed in Figures 3 and 4 were computed without detailing which of the three explicit sugar molecules included in each simulation shows the differential behavior. Next, an extensive study of the characteristics of the interaction of both sugar types with the $\mathrm{CP}$ is presented for each of the three explicit molecules included in the simulation box. 
Figure 5a summarizes the total time of interaction of each explicit glucose and fructose molecule with the electroactive polymer. Apparently, fructose seems to present a greater propensity to interact with the $\mathrm{CP}$ than glucose. Thus, the total time of interaction averaged for glucose and fructose is 2.76 and $6.13 \mathrm{~ns}$, respectively. Analysis of the mean residence time, which is defined as the average time a sugar molecule spends forming specific hydrogen bonding interactions with PHMeDOT chains without disruption, drastically reduces such difference, even though the ranking is preserved (Figure 5b). Thus, the mean residence time for glucose and fructose is 1.37 and $2.27 \mathrm{ps,}$ respectively. However, the scenario changes when the population of all possible specific interactions is examined. Figure 6 displays the distribution of specific interactions for each explicit sugar molecule, expressed as population in percentage of (HMeDOT)OM $\cdots \mathrm{H}-\mathrm{O}\left(\right.$ sugar), (HMeDOT)OM-H $\cdots \mathrm{O}$ (sugar) and (HMeDOT)OE $\cdots \mathrm{H}_{-}$ $\mathrm{O}$ (sugar) hydrogen bonds.

Results reveal that when fructose interacts with the $\mathrm{CP}$, it does preferentially through an undefined mixture of interactions. Thus, fructose acts either as hydrogen bond acceptor networking with the exocyclic hydroxyl group of the PHMeDOT repeat units or as hydrogen bond donor approaching the oxygen atoms of the PHMeDOT dioxane rings. Besides, only one of three fructose molecules (labeled as \# 1 in Figure 6) is able to interact with the exocyclic OM atom of PHMeDOT repeat units as hydrogen bond acceptor, even though it is the molecule that interacts lesser time with the CP. Moreover, in this particular case, such fructose molecule presents the longest averaged residence time over a PHMeDOT repeat unit among the three explicit sugar molecules.

The scenario depicted by glucose is quite opposite to the behavior of fructose. Although glucose molecules present lower mean residence times and total interaction times than fructose molecules, when they interact with the CP clearly prefer with the 
exocyclic hydroxyl group. It is worth noting that this is the only chemical group that differentiates this polymer from PEDOT, which is not able to detect glucose without the assistance of the GOx enzyme. ${ }^{31,49}$ Moreover, glucose prefers to interact as hydrogen bond donor using the hydroxyl groups tether to the main sugar ring that doing it as acceptor via oxygen $\mathrm{O} 5$ (i.e. analogous to the oxygen $\mathrm{O} 6$ in the fructose case). This interaction is illustrated in Figure 6d, which represents two close glucose molecules forming a network with specific $\mathrm{OE} \cdots \mathrm{H}-\mathrm{O}$ and $\mathrm{OM} \cdots \mathrm{H}-\mathrm{O}$ interactions. Overall, MD simulations show that glucose forms a well-defined pattern of specific hydrogen bonding interactions that fructose does not present, which clearly makes understandable the experimental observations.

\section{CONCLUSIONS}

The ability of PHMeDOT to recognize glucose and fructose at the molecular level has been examined by classical MD simulations, evaluating the formation of PHMeDOT ${ }^{\cdots}$ sugar specific interactions. MD simulations on detailed molecular models, which involved the PHMeDOT chains of a film electrochemically deposited onto a stainless steel substrate and explicit sugar molecules in aqueous environment, indicate that the affinity between PHMeDOT and glucose is very well-defined. Thus, this is dominated by specific hydrogen bonding interactions in which glucose acts as hydrogen bond donor using the hydroxyl groups and the $\mathrm{OE}$ and $\mathrm{OM}$ oxygen atoms of PHMeDOT behave as hydrogen bond acceptors. In contrast, PHMeDOT $\cdots$ fructose interactions are much less defined and rarely involve the $\mathrm{OM}$ atom of the exocyclic hydroxymethyl group, which is the distinctive chemical feature of PHMeDOT when compared to other CPs that do not behave as glucose sensors. Electrochemical assays are fully consistent with these results, indicating that PHMeDOT selectively 
discriminate glucose from fructose. Overall, results presented in this work allows us to conclude that to design new non-enzymatic polymeric sensors with a large capacity of detecting glucose, we should focus on CPs able to reinforce the formation of specific hydrogen bonding interactions with such sugar.

\section{ACKNOWLEDGEMENTS}

Authors acknowledge MINECO-FEDER (MAT2015-69367-R) and the Agència de Gestió d'Ajuts Universitaris i de Recerca (2017SGR359) for financial support. Support for the research of C.A. was received through the prize "ICREA Academia" for excellence in research funded by the Generalitat de Catalunya.

\section{REFERENCES}

1. K. Ogurtsova, J. D. D. R. Fernandes, Y. Huang, U. Linnenkamp, L. Guariguata, N. H. Cho, D. Cavan, J. E. Shaw and L. E. Makaroff, Diabetes Res. Clin. Pract., 2017, 128, 40 .

2. B. E. Lewis, N. Choytun, V. L. Schramm and A. J. Bennett, J. Am. Chem. Soc., 2006, 128, 5049.

3. B. Beden, F. Largeaud, K. B. Kokoh and C. Lamy, Electrochim. Acta, 1996, 41, 701.

4. A. A. Rossini and J. S. Soeldner, J. Clin. Invest., 1976, 57, 1083.

5. S. A. Zaidi and J. H. Shin, Talanta, 2016, 149, 30.

6. R.-J. Chung, A.-N. Wang and S.-Y Peng, Nanomaterials, 2017, 7, 39.

7. B. K. Shrestha, R. Ahmad, S. Shrestha, C. H. Park and C. S. Kim, Sci. Rep., 2017, 7, 1691 . 
8. S. Kumar-Krishnan, M. G. F. Garcia, E. Prokjorov, M. Estevez-Gonzlález, R. Perez, R. Esparza and M. Mayyappan. J. Mater. Chem. B., 2017, 5, 7072.

9. J. T. Holland, C. Lau, S. Brozik, P. Attanassov and S. Banta, J. Am. Chem. Soc., 2011, 133, 19262.

10. C. Alemán, G. Fabregat, E. Armelin, J. J. Buendía and J. Llorca, J. Mater. Chem. B, $2018,6,6515$.

11. S. Ferri, K. Kojima and K. Sode, J. Diabetes. Sci. Technol., 2011, 5, 1068.

12. S. K. Vashist, D. Zheng, K. Al-Rubeaan, J. H. T. Luong and F. S. Sheu, Anal. Chim. Acta, 2011, 703, 124.

13. S. Y. Tee, C. Teng and E. Ye, Mater. Sci. Eng. C, 2017, 70, 1018.

14. H. Zhu, L. Li, W. Zhou, Z. P. Shao and X. J. Chen, J. Mater. Chem. B, 2016, 4, 7333.

15. G. Wang, X. He, L L. Wang, A. X. Gu, Y. Huang, B. Fang, B. Y. Geng and X. J. Zhang, Microchim. Acta, 2013, 180, 161.

16. P. Si, Y. J. Huang, T. H. Wang and J. M. Ma, RSC Adv., 2013, 3, 3487-3502.

17. S. Park, S. Park, R. A. Jeong, H. Boo, J. Park, H. C. Kim and T. D. Chung, Biosens. Bioelectron., 2012, 31, 284.

18. A. Weremfo, S. T. C. Fong, A. Khan, D. B. Hibbert and C. Zhao, Electrochim. Acta, 2017, 231, 20.

19. H. H. Shu, L. L. Cao, G. Chang, H. P. He, Y. T. Zhang and Y. B. He, Electrochim. Acta, 2014, 132, 524-.

20. G. Chang, H. H. Shu, K. Ji, M. Oyama, X. Liu and Y. B. He, Appl. Surf. Sci., 2014, 288, 524.

21. S. Darvishi, M. Souissi, F. Karimzadeh, M. Kharaziha, R. Sahara and S. Ahadian, Electrochim. Acta, 2017, 240, 388. 
22. S. F. Fu, C. Z. Zhu, J. H. Song, M. Engelhard, H. B. Xia, D. Du and Y. H. Lin, ACS Appl. Mater. Interfaces, 2016, 8, 22196.

23. Y. Zhao, L. Z. Fan, B. Hong, J. L. Ren, M. S. Zhang, Q. M. Que, J. Y. Ji, Sens. Actuators B Chem., 2016, 231, 800.

24. L. Wang, Y. Y. Zhang, J. Yu, J. He, H. Yang, Y. H. Ye and Y. H. Song, Sens. Actuators B Chem., 2017, 239, 172.

25. D. M. Kim, J. M. Moon, W. C. Lee, J. H. Yoon, C. S. Choi and Y. B. Shim, Biosens. Bioelectron., 2017, 91, 276.

26. Y. Q. Yang, C. L. Yi, J. Luo, R. Liu, J. K. Liu, J. Q. Jiang and X. Y. Liu, Biosens. Bioelectron., 2011, 26, 2607.

27. M. C. D. Cooray, Y. Liu, S. J. Langford, A. M. Bond and J. Zhang, Anal. Chim. Acta, 2015, 856, 27.

28. H. Çiftçi and U. Tarmer, React. Funct. Polym., 2012, 72, 127.

29. Z. Shahnavaz, F. Lorestani, Y. Alias and P. M. Woi, Appl. Surf. Sci., 2014, 317, 622.

30. H. Çiftçi and U. Tarmer, Electrochim. Acta, 2013, 90, 358.

31. M. A. Hocevar, G. Fabregat, E. Armelin, C. A. Ferreira and C. Alemán, Eur. Polym. J., 2016, 79, 132.

32. C. Ocampo, R. Oliver, E. Armelin, C. Alemán and F. Estrany, J. Polym. Res., 2006, 13, 193.

33. P. Cieplak, W. Cornell, C. I. Bayly and P. A. Kollman, J. Comput. Chem., 1995, 16, 1357.

34. J. Preat, D. Zanuy, E. A. Perpete and C. Aleman, Biomacromolecules, 2011, 12, 1298. 
35. W. D. Cornell, P. Cieplak, C. I. Bayly, I. R. Gould, K. M. Merz, D. M. Ferguson, D. C. Spellmeyer, T. Fox, J. W. Caldwell and P. A. Kollman, J. Am. Chem. Soc., $1995, \mathbf{1 1 7}, 5179$.

36. D. Zanuy and C. Aleman, Soft Matter, 2013, 9, 11634.

37. K. N. Kirschner, A. B. Yongye, S. M. Tschampel, C. R. Daniels, B. L. Foley and R. J. Woods, J. Comput. Chem., 2008, 29, 622.

38. W. L. Jorgensen, J. Chandrasekhar, J. D. Madura, R. W. Impey and M. L. Klein, J. Chem. Phys., 1983, 79, 926.

39. D. Zanuy, G. Fabregat, J. Triguero and C. Alemán, J. Phys. Chem. C, 2018, 122, 20261.

40. D. Curcó, D. Zanuy, R. Nussinov and C. Aleman, J. Comput. Chem., 2011, 32, 607.

41. J. C. Phillips, R. Braun, W. Wang, J. Gumbart, E. Tajkhorshid, E. Villa, C. Chipot, R. D. Skeel, L. Kale and K. Schulten, J. Comput. Chem., 2005, 26, 1781.

42. H. J. C. Berendsen, J. P. M. Postma, W. F. van Gunsteren, A. DiNola and J. R. Haak, J Chem Phys, 1984, 81, 3684.

43. G. J. Martyna, D. L. Tobia and M. L. Klein, J. Chem. Phys., 1994, 101, 4177.

44. S. E. Feller, Y. Zhang, E. W. Pastor and B. R. Brooks, J. Chem. Phys., 1995, 103, 4613.

45. S. Toxvaerd, J. Chem. Phys., 1990, 93, 4290.

46. C. L. Wennberg, T. Murtola, B. Hess and E. Lindahl, J. Chem. Theory Comput., 2013, 9, 3527.

47. A. Toukmaji, C. Sagui, J. Board and T. Darden, J. Chem. Phys., 2000, 113, 10913.

48. J. Wang, Chem. Rev., 2008, 108, 814.

49. J. J. Buendía, G. Fabregat, A. Castedo, J. Llorca and C. Alemán, Plasma Process. Polym., 2018, 15, 1700133. 


\section{CAPTIONS TO FIGURES}

Figure 1. Current-time plot for PHMeDOT upon: (a) the successive addition of 1 $\mathrm{mM}$ glucose in $0.1 \mathrm{M}$ PBS; and (ii) the successive addition in $0.1 \mathrm{M}$ PBS of: (i) $1 \mathrm{mM}$ glucose, $1 \mathrm{mM}$ fructose (three times) and $1 \mathrm{mM}$ glucose. Polarization potential: -600 $\mathrm{mV}$ vs $\mathrm{Ag} \mid \mathrm{AgCl}$.

Figure 2. Radial distribution of distances between the mass centers of any sugar molecule and the PHMeDOT repeat units located within a cutoff distance of $14 \AA$ (red line) or all $\mathrm{ClO}_{4}{ }^{-}$counterions (blue line) for (a) glucose and (b) fructose.

Figure 3. (a) Atomistic scheme of the molecular species investigated in this work showing the labels used to identify all oxygen atoms that may act as hydrogen bonding acceptors. Hydrogen atoms attached to such oxygen atoms correspond to the hydrogen bonding donors. Radial distributions of distances between any potential hydrogen bonding acceptor of (b) glucose and (c) fructose and the hydrogen bonding donor of PHMeDOT repeat units (i.e. the hydrogen atom attached to the exocyclic OM atom).

Figure 4. Radial distributions of distances between the dioxane hydrogen bonding acceptor of PHMeDOT (i.e. OE oxygen atom in Figure 3a) and the hydrogen bonding donors of the sugar (left), and between the exocyclic hydrogen bonding acceptor of PHMeDOT (i.e. OM oxygen atom in Figure 2a) and the hydrogen bonding donors of the sugar (right) for (a) glucose and (b) fructose.

Figure 5. Comparison of (a) the total time of interaction and (b) the mean residence time for each of the three glucose and fructose molecules included explicitly in the MD simulations. Average values are also represented.

Figure 6. Distributions of the specific interactions formed by each explicit glucose and fructose molecule included in MD simulation. Distributions are expressed as population in percentage of the following hydrogen bonds: (a) (HMeDOT)OM $\cdots \mathrm{H}-$ 
$\mathrm{O}$ (sugar); (b) (HMeDOT)OM-H $\cdots \mathrm{O}$ (sugar); and (c) (HMeDOT)OE $\cdots \mathrm{H}-\mathrm{O}$ (sugar). Average values are also represented. (d) Example of (HMeDOT)OE $\cdots \mathrm{H}-\mathrm{O}$ (glucose) hydrogen bonding interactions (dashed pink lines), which dominate the recognition pattern of the $\mathrm{CP}$ sensor. 
(a)

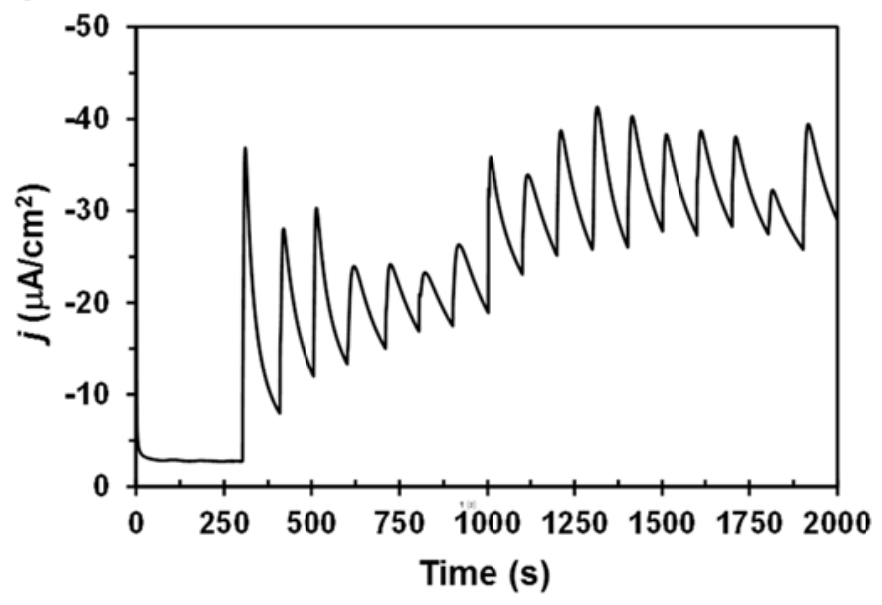

(b)

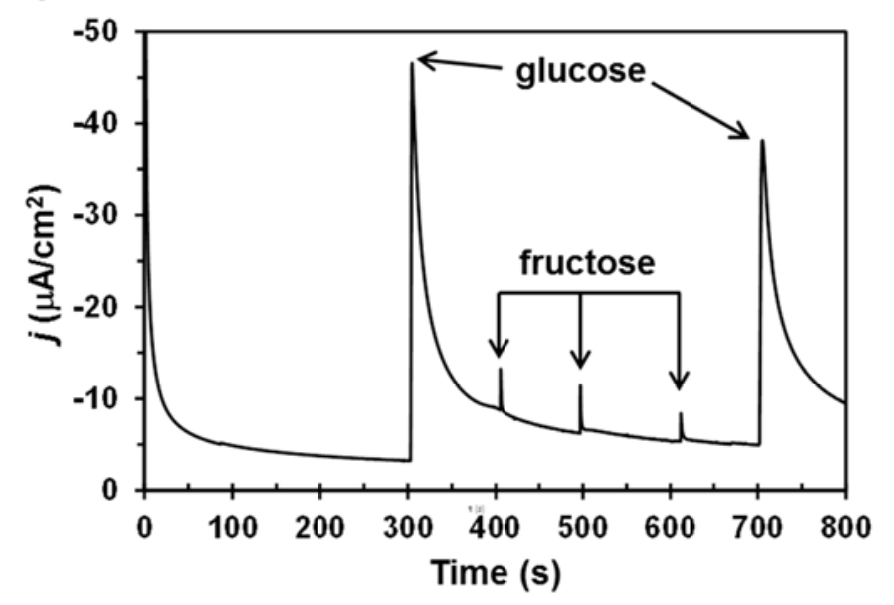

Figure 1 

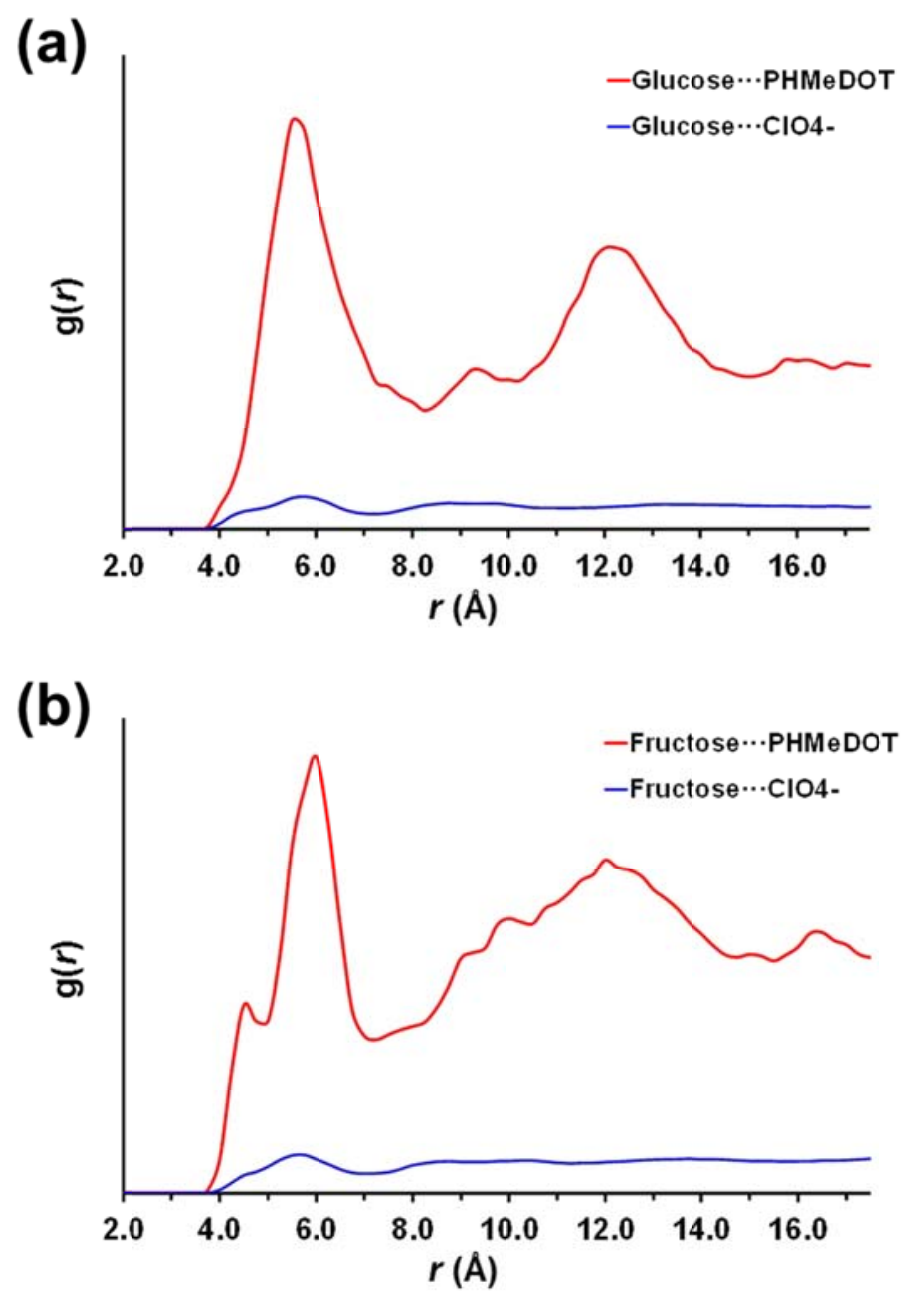

Figure 2 
(a)
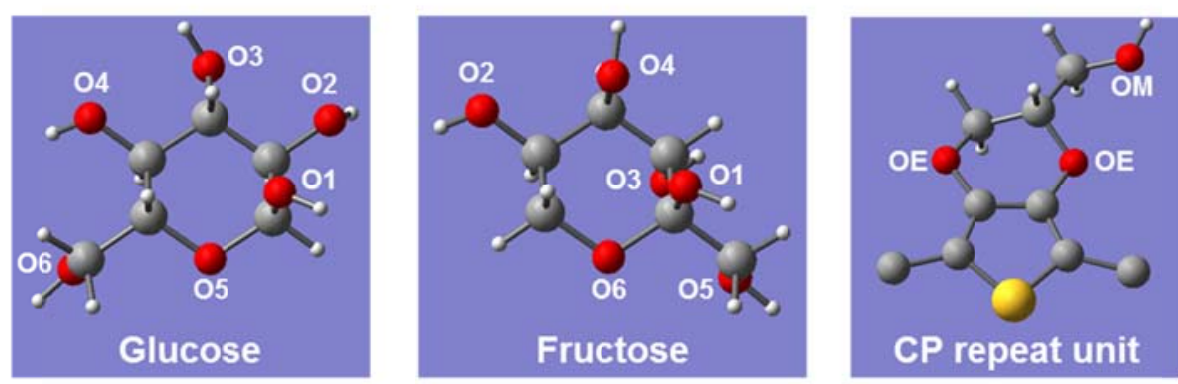

(b)

(c)
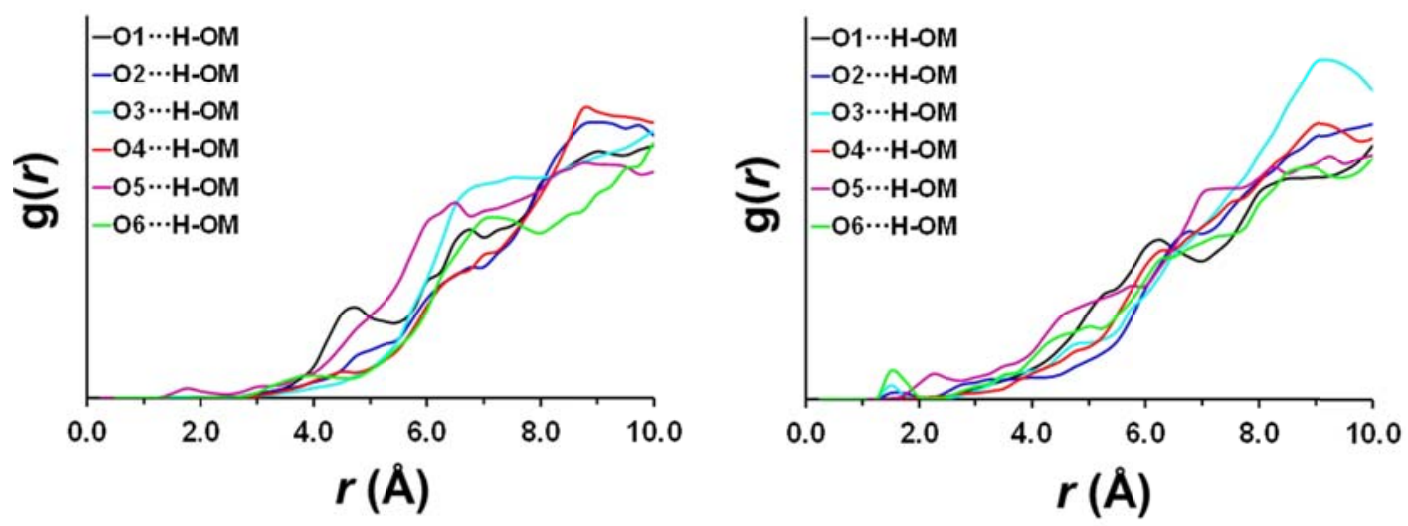

Figure 3 
(a)
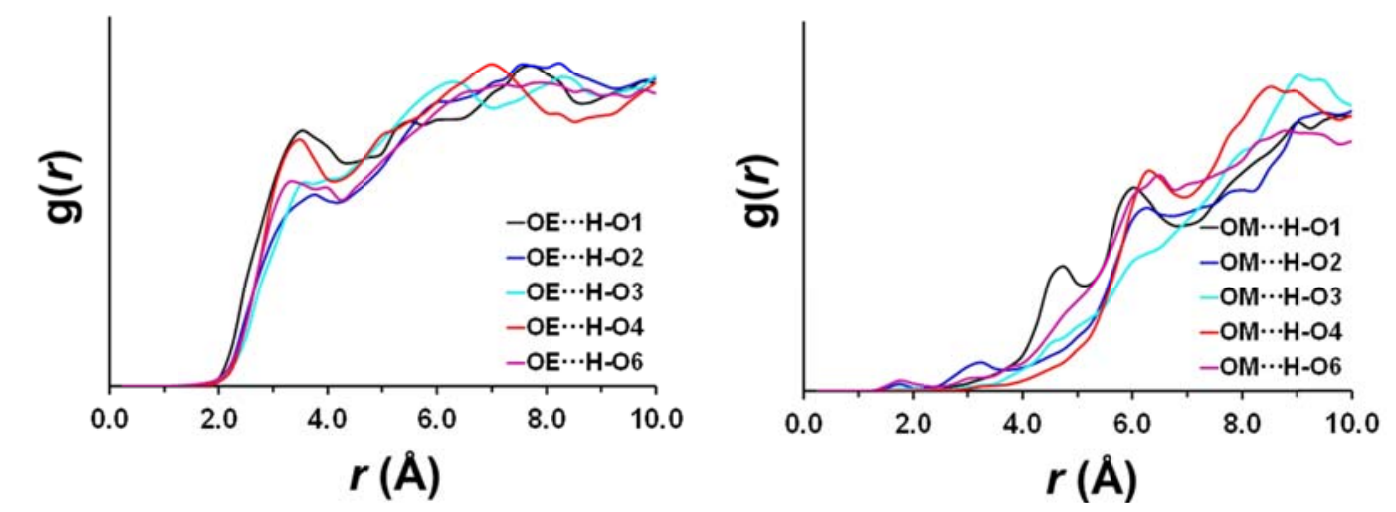

(b)
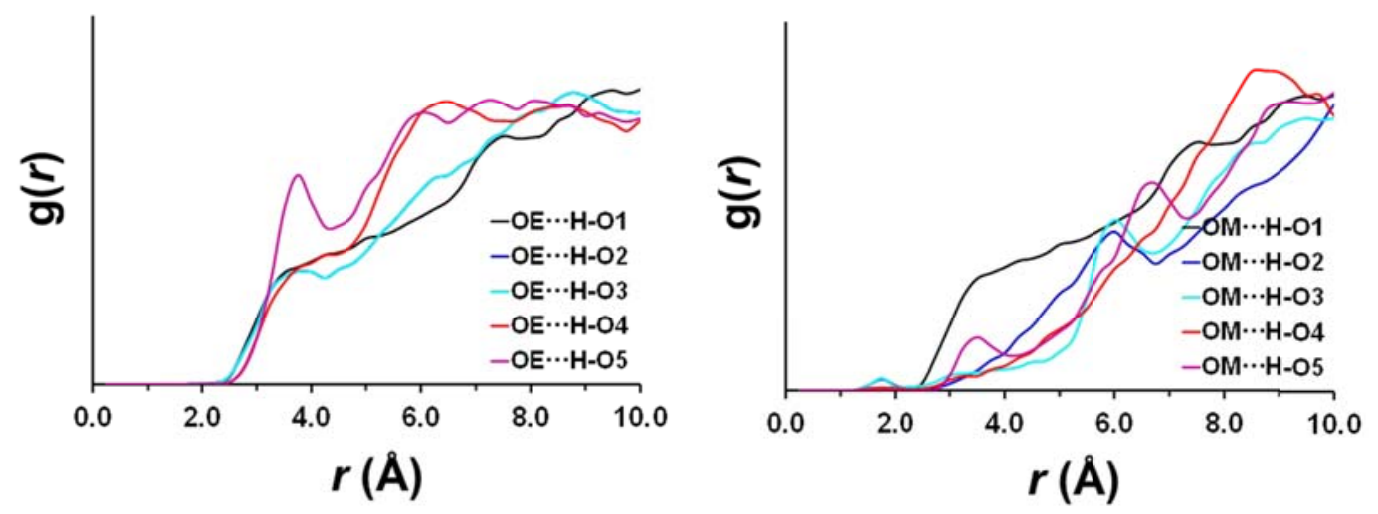

Figure 4 
(a)

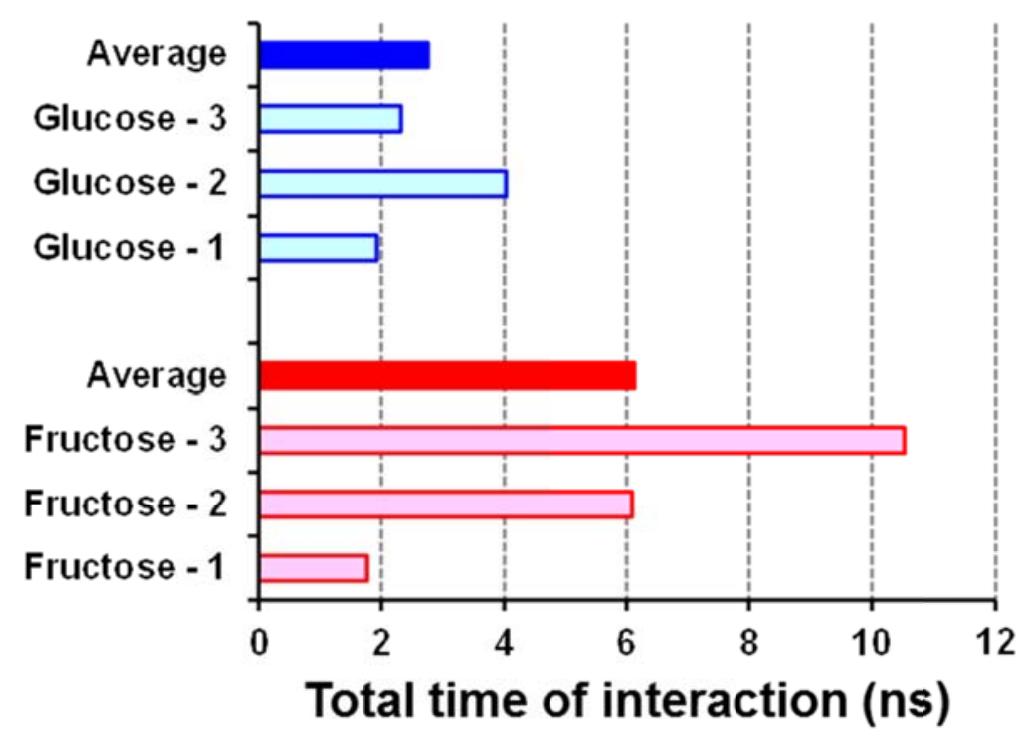

(b)

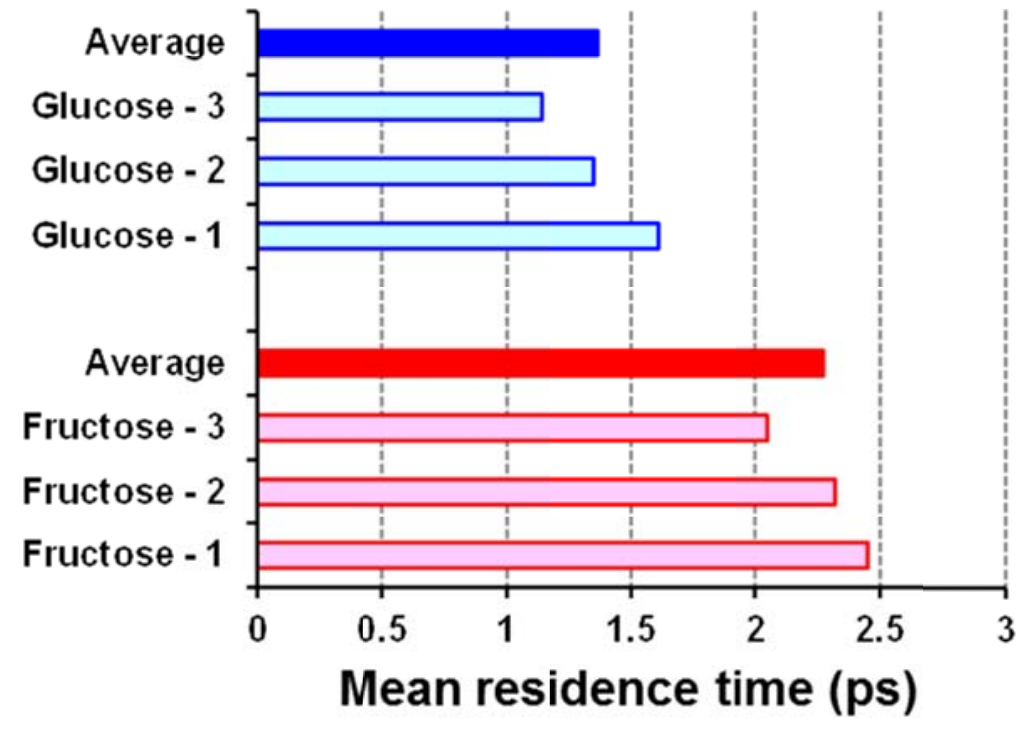

Figure 5 
(a)

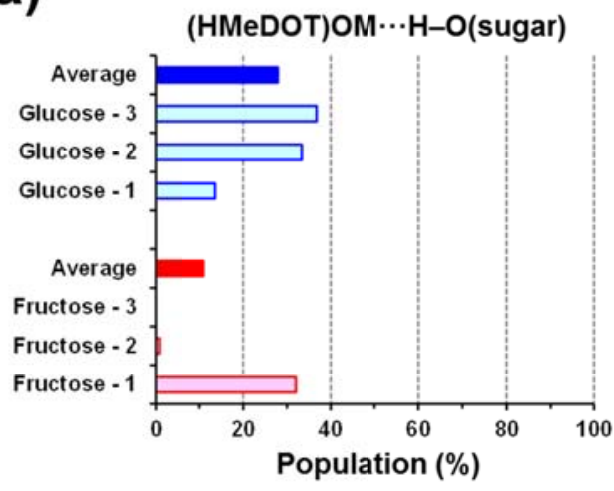

(b)

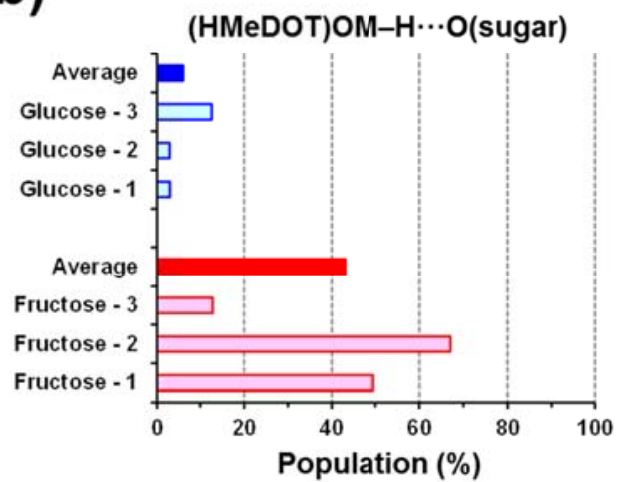

(c)

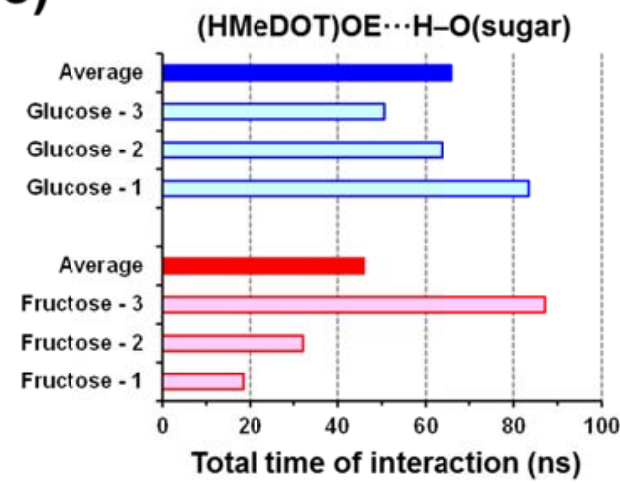

(d)

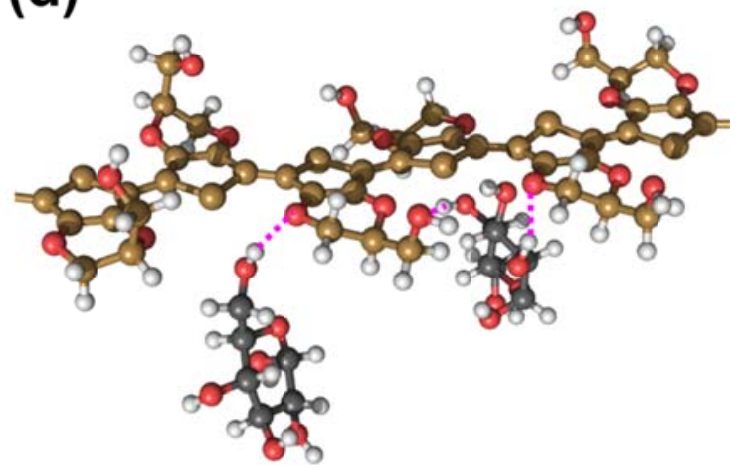

Figure 6 
GRAPHICAL ABSTRACT

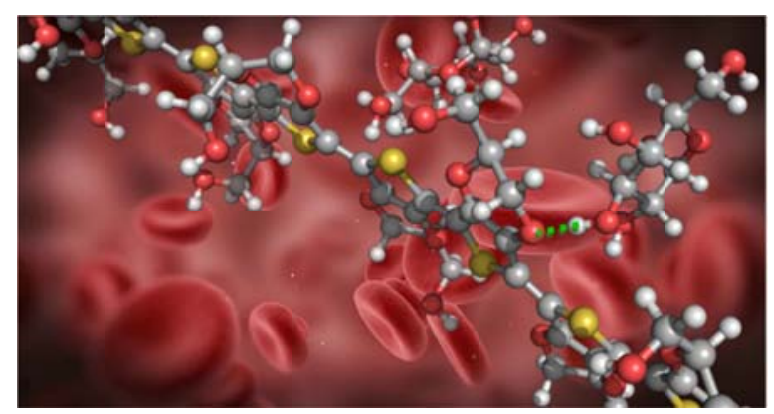

